\title{
Thermal Effects on Vibration and Control of Piezocomposite Kirchhoff Plate Modeled by Finite Elements Method
}

\author{
M. Sanbi, ${ }^{1}$ R. Saadani, ${ }^{2}$ K. Sbai, ${ }^{2}$ and M. Rahmoune ${ }^{2}$ \\ ${ }^{1}$ Equipe Sciences et Technologies Avancées, Ecole Nationale des Sciences Appliquées, Université Abdelmalek Essaadi, \\ 93030 Tétouan, Morocco \\ ${ }^{2}$ Laboratoire d'Etude des Matériaux Avancés et Applications, Faculté des Sciences et Ecole Supérieure de Technologie, \\ Université Moulay Ismail, 50040 Meknès, Morocco \\ Correspondence should be addressed to M. Rahmoune; rahmoune@umi.ac.ma
}

Received 5 December 2014; Revised 2 April 2015; Accepted 3 April 2015

Academic Editor: Weihua Li

Copyright (C) 2015 M. Sanbi et al. This is an open access article distributed under the Creative Commons Attribution License, which permits unrestricted use, distribution, and reproduction in any medium, provided the original work is properly cited.

\begin{abstract}
Theoretical and numerical results of the modeling of a smart plate are presented for optimal active vibration control. The smart plate consists of a rectangular aluminum piezocomposite plate modeled in cantilever configuration with surface bonded thermopiezoelectric patches. The patches are symmetrically bonded on top and bottom surfaces. A generic thermopiezoelastic theory for piezocomposite plate is derived, using linear thermopiezoelastic theory and Kirchhoff assumptions. Finite element equations for the thermopiezoelastic medium are obtained by using the linear constitutive equations in Hamilton's principle together with the finite element approximations. The structure is modelled analytically and then numerically and the results of simulations are presented in order to visualize the states of their dynamics and the state of control. The optimal control LQGKalman filter is applied. By using this model, the study first gives the influences of the actuator/sensor pair placement and size on the response of the smart plate. Second, the effects of thermoelastic and pyroelectric couplings on the dynamics of the structure and on the control procedure are studied and discussed. It is shown that the effectiveness of the control is not affected by the applied thermal gradient and can be applied with or without this gradient at any time of plate vibrations.
\end{abstract}

\section{Introduction}

In the piezoelectric sensors applications, mechanically or thermally induced deformations can be determined from measurement of the induced electrical potential, whereas in piezoelectric actuator applications deformation of strain can be controlled through the introduction of appropriate electric potential. By integrating distributed piezoelectric sensors/actuators and advanced composites, the potential exists for forming high-strength, high stiffness, lightweight structures capable of self-monitoring and self-controlling. Typical applications of such structures are envisioned in the thermal distortion management of propulsion components and space structures. Before they can be utilized in these applications, the performance of piezoelectric structures in thermal environment must be quantified. Thermal effects become important when the piezoelectric structure has to operate in either extremely hot or cold temperature environments. These extreme conditions may severely affect the response of piezoelectric elements by induction of thermal stresses resulting from thermoelastic and pyroelectric coefficients.

Recently, there have been various mathematical models developed to describe the behavior of the piezocomposite or laminated plates actuated and sensed by piezoelectric materials. Görnandt and Gabbert [1] have analysed the finite element method of thermopiezoelectric smart structures. Vel and Batra [2] presented a generalized plane strain thermopiezoelectric analysis of multilayered plates. de Abreu et al. [3] have implemented finite element modeling of a plate with localized piezoelectric sensors and actuators. Deü and Benjeddou [4] have studied free-vibration analysis of laminated plates with embedded shear-mode piezoceramic layers. Tanaka and Sanada [5] have detailed modal control of a rectangular plate using smart sensors and smart actuators. 
TABLE 1: Properties of the plate, sensor, and actuator.

\begin{tabular}{|c|c|c|c|}
\hline \multirow[t]{2}{*}{ Properties/labels/units } & Plate & Sensor & $\begin{array}{c}\text { Actuator } \\
\text { PZT-5H32 }\end{array}$ \\
\hline & $\mathrm{Al}$ & PVDF & PZT-5H32 \\
\hline Length $L(\mathrm{~m})$ & 0.6 & 0.25 & 0.25 \\
\hline Width $l(\mathrm{~m})$ & 0.4 & 0.25 & 0.25 \\
\hline Thickness $t(\mathrm{~m}) \times 10^{-3}$ & 1 & 0.2 & 0.2 \\
\hline Density $\rho\left(\mathrm{g} / \mathrm{cm}^{3}\right)$ & 8.03 & 1.78 & 7.7 \\
\hline Young modulus $E(\mathrm{GPa})$ & 68 & 5.04 & 62 \\
\hline \multicolumn{4}{|l|}{ Reduced elastic constants (GPa) } \\
\hline $\bar{c}_{11}^{E}$ & - & 5.04 & 66.2 \\
\hline $\bar{c}_{12}^{E}$ & - & 3.25 & 19.2 \\
\hline $\bar{c}_{66}^{E}$ & - & - & 23.5 \\
\hline $\begin{array}{l}\text { Piezoelectric stress constant } \\
g_{31}(\mathrm{Vm} / \mathrm{N})\end{array}$ & - & 0.15 & -9.11 \\
\hline $\begin{array}{l}\text { Piezoelectric strain constant } \\
d_{31} \times 10^{-12}(\mathrm{~m} / \mathrm{V})\end{array}$ & - & 4.34 & -274 \\
\hline $\begin{array}{l}\text { Reduced piezoelectric constant } \\
\bar{e}_{31}\left(\mathrm{C} / \mathrm{m}^{2}\right)\end{array}$ & - & 0.018 & -23.4 \\
\hline $\begin{array}{l}\text { Pyroelectric constant } \\
p \times 10^{-5} \mathrm{Cm}^{-2} \mathrm{~K}^{-1}\end{array}$ & - & -1.25 & - \\
\hline $\begin{array}{l}\text { Dielectric constant } \epsilon_{33}^{S} / \epsilon_{0} \\
\left(\epsilon_{0}=8.85410^{-12} \mathrm{~F} / \mathrm{m}\right)\end{array}$ & - & 12 & 3300 \\
\hline $\begin{array}{l}\text { Thermal conductivity } \\
K_{d}(\mathrm{~W} / \mathrm{m} \cdot \mathrm{K})\end{array}$ & - & 1.5 & 1.5 \\
\hline $\begin{array}{l}\text { Thermal expansion } \\
\alpha \times 10^{-6}\left(\mathrm{~K}^{-1}\right)\end{array}$ & - & 140 & 3 \\
\hline Specific heat $c_{0} \mathrm{~J} \cdot \mathrm{kg}^{-1} \cdot \mathrm{K}^{-1}$ & - & 350 & 1.5 \\
\hline Damping $\alpha \times 10^{-3}$ & 1 & - & - \\
\hline Constants $\beta \times 10^{-3}$ & 0.1 & - & - \\
\hline
\end{tabular}

Trindade and Benjeddou [6] have discussed the critical evaluation and optimization of the effective electromechanical coupling coefficients of piezoelectric adaptive structures. Sanbi et al. [7] have analyzed thermoelastic and pyroelectric couplings effects on dynamics and active control of piezolaminated beam. A general solution for piezothermoelasticity of transversely isotropic piezoelectric materials and its applications have been deduced by Ding et al. [8].

In this work, fundamental equations governing the behavior of smart structures are at first given, and, based on the work of de Abreu et al. [3], the generalized linear finite element formulation of the problem is described. Thermopiezoelastic characteristics of aluminium rectangular plate and the theory of active structures in sensing and optimal control are established. A generic thermopiezoelastic theory for piezocomposite plate is derived, using linear thermopiezoelasticity and Kirchhoff assumptions. Generalized finite element equations for the thermopiezoelasticity are obtained by using the linear constitutive equations in Hamilton's principle together with the finite element approximations. A Kirchhoff four-node rectangular element with one electrical, one temperature, and three mechanical degrees of freedom is considered. The structure consists of a modeling of cantilevered piezocomposite plate with perfectly surface bonded thermopiezoelectric elements. The structure is modelled analytically and then numerically and the results of simulations are presented in order to visualize the states of their dynamics and the state of control. The optimal control LQG-Kalman accompanied is applied. The effects of thermoelastic and pyroelectric couplings coefficients on the dynamics of the structure and on the control effectiveness are discussed. We show that the control procedure cannot be perturbed by applying a thermal gradient and the control can be applied at any time during the period of vibration of the plate.

\section{Basic Equations and FEM Implementation}

The physical and dimensional characteristics of the plate material are given in Table 1. Properties for the two piezoelectric materials used in the simulation can be found in the literature as in $[9,10]$. The piezoelectric and elastic properties of the sensor are chosen smaller compared to those of the actuator. This is justified by the fact that the sensor must have high sensitivity to capture the deformation of the structure, while the actuator is required to act as a secondary source on the plate to deaden its vibrations. In order to model the structure 


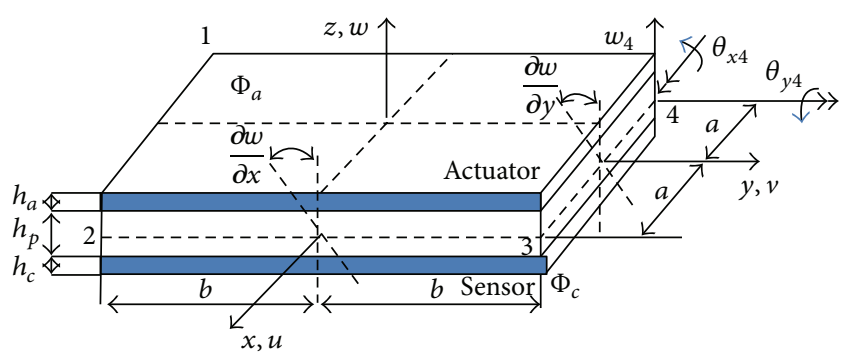

FIGURE 1: The finite element type "plate" used in the formulation and different degrees of freedom.

by finite element method, we consider an element with fournode quadrilateral flexible element type "plate." Each node has three mechanical degrees of freedom (dofs), the displacement in $z$-direction and two rotations in $x$ - and $y$-direction, one electric $\operatorname{dof} \Phi$, and one temperature $\operatorname{dof} \Theta$ (Figure 1).

In the present formulation, we assume that the piezoelectric layers are perfectly bonded and the formulation is restricted to linear elastic material behavior with small displacement and strains. This formulation uses the Kirchhoff assumption for thin plate in which the transverse normal remains straight and normal to the midsurface after deformation. The thickness of the plate does not change during a deformation. Depending on these assumptions and as shown in Figure 1, displacements $u, v$, and $w$ can be expressed by

$$
\begin{aligned}
& u=-z \frac{\partial w}{\partial x}, \\
& v=-z \frac{\partial w}{\partial y}, \\
& w=w(x, y),
\end{aligned}
$$

where $x$ and $y$ are the longitudinal and transverse axes located on the medial surface of the plate and $z$ is the thickness direction (Figure 1). The displacements $u, v$, and $w$ are taken along the axes $x, y$, and $z$, respectively. Assuming that the transverse deformation is negligible, the deformation in terms of displacements can be written as

$$
\varepsilon=\left[\begin{array}{lll}
\varepsilon_{x} & \varepsilon_{y} & \varepsilon_{x y}
\end{array}\right]^{T}=-z\left[\begin{array}{lll}
\frac{\partial^{2} w}{\partial x^{2}} & \frac{\partial^{2} w}{\partial y^{2}} & \frac{\partial^{2} w}{\partial x \partial y}
\end{array}\right]^{T} .
$$

For an isotropic material, the constraints are related to deformation by

$$
\sigma=[\mathscr{C}]\{\varepsilon\},
$$

where $\sigma=\left[\begin{array}{lll}\sigma_{x} & \sigma_{y} & \tau_{x y}\end{array}\right]^{T}$ and the matrix $\mathscr{C}$ is given by

$$
\mathscr{C}=\frac{E_{P}}{1-v^{2}}\left[\begin{array}{ccc}
1 & v & 0 \\
\nu & 1 & 0 \\
0 & 0 & \frac{(1-v)}{2}
\end{array}\right],
$$

with $v$ being the Poisson coefficient and $E_{P}$ the plate Young modulus.

In the finite element modeling, a four-node bending plate rectangular element based on the classical theory of plates is considered [11]. Each node element has three mechanical degrees of freedom: the displacement $\bar{w}$ in $z$-direction, $x$ rotation $\bar{\theta}_{y}$, and $y$-rotation $\bar{\theta}_{x}$ (Figure 1). The function of the displacement $w$ can be written as

$$
\begin{aligned}
w\left(x_{i}, y_{i}\right)= & c_{1}+c_{2} x_{i}+c_{3} y_{i}+c_{4} x_{i}^{2}+c_{5} x_{i} y_{i}+c_{6} y_{i}^{2} \\
& +c_{7} x_{i}^{3}+c_{8} x_{i}^{2} y_{i}+c_{9} x_{i} y_{i}^{2}+c_{10} y_{i}^{3}+c_{11} x_{i}^{3} y_{i} \\
& +c_{12} x_{i} y_{i}^{3},
\end{aligned}
$$

with

$$
\begin{aligned}
& i=1 \cdots 4 ; \\
& x_{1}=-a ; y_{1}=-b ; x_{2}=a ; y_{2}=b ; \\
& x_{3}=a ; y_{3}=b ; x_{4}=-a ; y_{4}=-b .
\end{aligned}
$$

The transverse displacement can be expressed by

$$
w=[P]^{T}[c],
$$

where

$$
\begin{aligned}
& {[c]=\left[\begin{array}{lllllll}
c_{1} & c_{2} & c_{3} & c_{4} & c_{5} & \cdots & c_{12}
\end{array}\right],} \\
& {[P]} \\
& =\left[\begin{array}{llllllllllll}
1 & x & y & x^{2} & x y & y^{2} & x^{3} & x^{2} y & x y^{2} & y^{3} & x^{3} y & x y^{3}
\end{array}\right]^{T} .
\end{aligned}
$$

We define the vector $q_{(i)}$ that contains the nodal displacements of the rectangular element by

$$
\begin{aligned}
& {\left[q_{i}\right]} \\
& =\left[\begin{array}{llllllllllll}
\bar{w}_{1} & \bar{\theta}_{x 1} & \bar{\theta}_{y 1} & \bar{w}_{2} & \bar{\theta} x_{2} & \bar{\theta}_{y 2} & \bar{w}_{3} & \bar{\theta}_{x 3} & \bar{\theta}_{y 3} & \bar{w}_{4} & \bar{\theta}_{x 4} & \bar{\theta}_{y 4}
\end{array}\right]^{T},
\end{aligned}
$$

where

$$
\begin{aligned}
\bar{w}_{i} & =\left.w\right|_{x_{i}, y_{i}}, \\
\bar{\theta}_{x_{i}} & =\left.\frac{\partial w}{\partial y}\right|_{x_{i}, y_{i}}, \\
\bar{\theta}_{y_{i}} & =\left.\frac{\partial w}{\partial y}\right|_{x_{i}, y_{i}} .
\end{aligned}
$$

By combining (5) and (8) with (9) for the four nodes, we obtain the following matrix expression:

$$
\left[q_{i}\right]=[X][c],
$$

where $[X]$ is a $(12 \times 12)$ matrix given by 


$$
[X]=\left[\begin{array}{cccccccccccc}
1 & x_{1} & y_{1} & x_{1}^{2} & x_{1} y_{1} & y_{1}^{2} & x_{1}^{3} & x_{1}^{2} y_{1} & x_{1} y_{1}^{2} & y_{1}^{3} & x_{1}^{3} y_{1} & x_{1} y_{1}^{3} \\
0 & 0 & 1 & 0 & x_{1} & 2 y_{1} & 0 & x_{1}^{2} & 2 x_{1} y_{1} & 3 y_{1}^{3} & x_{1}^{3} & 3 x_{1} y_{1}^{2} \\
0 & -1 & 0 & -2 x_{1} & -y_{1} & 0 & -3 x_{1}^{2} & 2 x_{1} y_{1} & -y_{1}^{2} & 0 & -3 x_{1}^{2} y_{1} & -y_{1}^{3} \\
1 & x_{1} & y_{1} & x_{1}^{2} & x_{1} y_{1} & y_{1}^{2} & x_{1}^{3} & x_{1}^{2} y_{1} & x_{1} y_{1}^{2} & y_{1}^{3} & x_{1}^{3} y_{1} & x_{1} y_{1}^{3} \\
0 & 0 & 1 & 0 & x_{1} & 2 y_{1} & 0 & x_{1}^{2} & 2 x_{1} y_{1} & 3 y_{1}^{3} & x_{1}^{3} & 3 x_{1} y_{1}^{2} \\
0 & -1 & 0 & -2 x_{1} & -y_{1} & 0 & -3 x_{1}^{2} & 2 x_{1} y_{1} & -y_{1}^{2} & 0 & -3 x_{1}^{2} y_{1} & -y_{1}^{3} \\
1 & x_{1} & y_{1} & x_{1}^{2} & x_{1} y_{1} & y_{1}^{2} & x_{1}^{3} & x_{1}^{2} y_{1} & x_{1} y_{1}^{2} & y_{1}^{3} & x_{1}^{3} y_{1} & x_{1} y_{1}^{3} \\
0 & 0 & 1 & 0 & x_{1} & 2 y_{1} & 0 & x_{1}^{2} & 2 x_{1} y_{1} & 3 y_{1}^{3} & x_{1}^{3} & 3 x_{1} y_{1}^{2} \\
0 & -1 & 0 & -2 x_{1} & -y_{1} & 0 & -3 x_{1}^{2} & 2 x_{1} y_{1} & -y_{1}^{2} & 0 & -3 x_{1}^{2} y_{1} & -y_{1}^{3}
\end{array}\right] .
$$

Therefore, the coefficient vector $[c]$ can be calculated from (10) as follows:

$$
[c]=[X]^{-1}\left\{q_{i}\right\} .
$$

Substituting (12) into (6) leads to

$$
w=\left[N_{w}\right] q_{i},
$$

where $\left[N_{w}\right]$ is the $z$-direction shape function matrix given by

$$
\left[N_{w}\right]=P^{T}[X]^{-1} .
$$

Substituting (3) into (2) yields

$$
[\varepsilon]=-z\left[\frac{\partial^{2}[P]^{T}}{\partial x^{2}} \frac{\partial^{2}[P]^{T}}{\partial y^{2}} 2 \frac{\partial^{2}[P]^{T}}{\partial x \partial y}\right]^{T}[X]^{-1}\left\{q_{i}\right\} .
$$

By manipulating (15), we can obtain

$$
[\varepsilon]=-z\left[L_{k}\right][X]^{-1}\left\{q_{i}\right\},
$$

with

$$
\begin{aligned}
& {\left[L_{k}\right]} \\
& \quad=\left[\begin{array}{cccccccccccc}
0 & 0 & 0 & 2 & 0 & 0 & 6 x & 2 y & 0 & 0 & 6 x y & 0 \\
0 & 0 & 0 & 0 & 0 & 2 & 0 & 0 & 2 x & 6 y & 0 & 6 x y \\
0 & 0 & 0 & 0 & 2 & 0 & 0 & 4 x & 4 y & 0 & 6 x^{2} & 6 y^{2}
\end{array}\right] .
\end{aligned}
$$

The displacements $u, v$, and $w$ can be expressed by the vector $\{q\}$ as

$$
\{q\}=\left\{\begin{array}{lll}
w & u & v
\end{array}\right\}^{T} .
$$

The substitution of (1) into (18) yields

$$
\{q\}=\left\{w-z \frac{\partial w}{\partial x}-z \frac{\partial w}{\partial y}\right\}^{T}
$$

and substituting (13) into (19), we get

$$
\{q\}=[H]\left[L_{M}\right]^{T}[X]^{-1}\left\{q_{i}\right\},
$$

where $\left[L_{M}\right]^{T}$ and $[H]$ are given by

$$
\begin{aligned}
& {\left[L_{M}\right]^{T}=\left\{\begin{array}{lll}
\{P\}^{T} & \frac{\partial\{P\}^{T}}{\partial x} & \frac{\partial\{P\}^{T}}{\partial y}
\end{array}\right\}^{T},} \\
& {[H]=\left[\begin{array}{ccc}
1 & 0 & 0 \\
0 & -z & 0 \\
0 & 0 & -z
\end{array}\right] \text {. }}
\end{aligned}
$$

Considering thermopiezoelastic material, the constitutive equations of linear thermopiezoelasticity and temperature are written as [12]

$$
\begin{aligned}
\{\sigma\} & =[C]^{E}\{\varepsilon\}-[e]\{E\}-[\lambda]\{\Theta\}, \\
\{D\} & =[e]^{T}\{\varepsilon\}-[\epsilon]^{S}\{E\}+[p]\{\Theta\}, \\
\{s\} & =[\lambda]^{T}\{\varepsilon\}+[p]^{T}\{E\}+[\widetilde{\alpha}]\{\Theta\},
\end{aligned}
$$

where the exponent $S$ means that the values are measured at constant strain and the exponent $E$ means they are measured at a constant electric field, $\{\sigma\}$ is the stress tensor, $\{D\}$ is the vector of electric displacement, $\{s\}$ is the entropy, $\{\Theta\}$ is the temperature vector, $\{\varepsilon\}$ is the tensor deformation, $\{E\}$ is the electric field, $[C]^{E}$ is the matrix of elastic coefficients at constant electric field, $[e]$ is the matrix of piezoelectric coefficients, $[\epsilon]^{S}$ is the tensor dielectric constant mechanical strain, $[p]$ is the pyroelectric tensor, and $[\lambda]$ is the thermoelastic tensor. $(\widetilde{\alpha})$ is the expansion coefficient given by $\widetilde{\alpha}=$ $\rho_{p} c_{0} / \Theta_{0}$, where $c_{0}$ and $\Theta_{0}$ are the specific heat and the initial temperature, respectively.

On the other hand, for the relation between $[e]$ and $[d]$, the coefficients of the piezoelectric strain are written as

$$
[e]=\left[C^{E}\right][d] .
$$

If the polarization direction is held in, the electrical potential applied or captured through the elements sensor or actuator is given by the following equation [13]:

$$
\Phi_{z}=\left(\frac{z-h_{p} / 2}{h}\right) \Phi
$$


where $h$ and $\Phi$ are the thickness and the maximum electric potential at the outer surface of the corresponding thermopiezoelectric element (actuator or sensor) and $z\left(z_{a}\right.$ or $\left.z_{c}\right)$ is defined in the following two intervals:

$$
\begin{aligned}
& \frac{h_{p}}{2} \leq z_{a} \leq \frac{h_{p}}{2}+h_{a}, \\
& \frac{h_{p}}{2} \leq z_{c} \leq \frac{h_{p}}{2}+h_{c} .
\end{aligned}
$$

Assuming that the electric field $E$ is constant along the thickness of the actuator and sensor, we can therefore write

$$
E=-\frac{d \Phi_{z}}{d z}=-B_{z} \Phi=-\frac{\Phi}{h} .
$$

Hamilton's principle is used here to derive finite element equations and is given by

$$
\int_{t_{1}}^{t_{2}}\left[\delta\left(T-U+W_{e}-W_{\text {th }}\right)+\delta W\right] d t=0,
$$

where $t_{1}$ and $t_{2}$ are two arbitrary instants, $T$ is the kinematic energy, $U$ is the energy potential, $W_{e}$ is the work due to electrical forces, and $W_{\text {th }}$ is the work due to thermal stresses. The total kinematic energy $T$ and energy potential $U$ are given by the following relations:

$$
\begin{aligned}
& T=\frac{1}{2} \int_{V} \rho\{\dot{q}\}^{T}\{\dot{q}\} d V, \\
& U=\frac{1}{2} \int_{V}\{\varepsilon\}^{T}\{\sigma\} d V-\int_{V}\{\varepsilon\}^{T}\{\lambda\}\{\Theta\} d V,
\end{aligned}
$$

with $\{\dot{q}\}$ being the derivative with respect to time $t$ and a volume element $d V$ given by

$$
d V=d V_{p}+d V_{a}+d V_{c},
$$

where the indexes $p, a$, and $c$ refer to the plate, the actuator, and the sensor, respectively, and $d V_{p}, d V_{a}$, and $d V_{c}$ are given by

$$
\begin{aligned}
& d V_{p}=\int_{-h_{p} / 2}^{h_{p} / 2} \int_{-b}^{b} \int_{-a}^{a} d x d y d z, \\
& d V_{a}=\int_{-h_{p} / 2}^{h_{p} / 2+h_{a}} \int_{-b}^{b} \int_{-a}^{a} d x d y d z, \\
& d V_{c}=\int_{-h_{p} / 2-h_{c}}^{h_{p} / 2} \int_{-b}^{b} \int_{-a}^{a} d x d y d z .
\end{aligned}
$$

The energy of electric forces and elementary virtual work produced by the volume forces $\left\{f_{v}\right\}$, surface forces $\left\{f_{A}\right\}$, and the applied electrical loads are written as

$$
\begin{aligned}
W_{e}= & \frac{1}{2} \int_{V}\left(\{E\}^{T}\{D\}+\{p\}\{\Theta\}\right) d V, \\
\delta W= & \int_{V}\{\delta q\}^{T}\left\{f_{v}\right\} d V+\int_{A}\{\delta q\}^{T}\left\{f_{A}\right\} d A \\
& -\int_{A} \delta \Phi \sigma_{q} d A,
\end{aligned}
$$

with $D$ being the electric displacement vector, $f_{v}$ the volume force, $f_{A}$ the surface force, and $\sigma_{q}$ the electric surface stress.

Substituting (3) and (22) into (31) and (23) into (34), respectively, leads to

$$
\begin{aligned}
U= & \frac{1}{2} \int_{V}\{\varepsilon\}^{T}\left[C^{E}\right]\{\varepsilon\}-\frac{1}{2} \int_{V}\{\varepsilon\}^{T}[e]\{E\} d V \\
& -\frac{1}{2} \int_{V}\{\varepsilon\}^{T}[\lambda]\{\Theta\} d V, \\
W_{e}= & \frac{1}{2} \int_{V}\{E\}^{T}[e]^{T}\{\varepsilon\}+\frac{1}{2} \int_{V}\{E\}^{T}[\epsilon]^{T}\{E\} d V \\
& +\frac{1}{2} \int_{V}\{E\}^{T}[p]^{T}\{\Theta\} d V .
\end{aligned}
$$

The substitution of (30), (35), and (36) into (29) gives

$$
\begin{aligned}
& \int_{t_{1}}^{t_{2}}\left[\int_{V} \rho\{\delta q\}^{T}\{\ddot{q}\} d V-\int_{V}\{\delta \varepsilon\}^{T}\left[C^{E}\right]\{\varepsilon\}\right. \\
& +\int_{V}\{\delta \varepsilon\}^{T}[e]^{T}\{E\} d V+\int_{V}\{\delta \varepsilon\}^{T}[\lambda]^{T}\{\Theta\} d V \\
& +\int_{V}\{\delta E\}^{T}[e]\{\varepsilon\} d V+\int_{V}\{\delta E\}^{T}[\epsilon]^{T}\{E\} d V \\
& \quad+\int_{V}\{\delta E\}^{T}[p]\{\Theta\} d V+\int_{V}\{\delta q\}^{T}\left\{f_{v}\right\} d V \\
& \left.\quad+\int_{V}\{\delta q\}^{T}\left\{f_{A}\right\} d V-\int_{V} \delta \Phi \sigma_{q} d A\right] d t=0
\end{aligned}
$$

Substituting (20), (16), and (28) into (37) leads to

$$
\begin{gathered}
\int_{t_{1}}^{t_{2}}\left[\{ \delta q _ { k } \} ^ { T } \left[\left[M_{q q}^{e}\right]\left\{\ddot{q}_{k}\right\}+\left[K_{q q}^{e}\right]\left\{q_{k}\right\}+\left[K_{q \Phi}^{e}\right]\{\Phi\}\right.\right. \\
\left.-\left[K_{q \Theta}^{e}\right]\{\Theta\}-\{\bar{f}\}\right]+\{\delta \Phi\}\left[\left[K_{\Phi q}^{e}\right]\left\{q_{k}\right\}\right. \\
\left.\left.+\left[K_{\Phi \Phi}^{e}\right]\{\Phi\}+\left[K_{\Phi \Theta}^{e}\right]\{\Theta\}+\left\{Q_{a}\right\}\right]\right] d t=0,
\end{gathered}
$$

where

$$
\begin{aligned}
& {\left[M_{q q}^{e}\right]=\rho \int_{V}[X]^{-T}\left[L_{M}\right][H]^{T}[H]\left[L_{M}\right]^{T}[X]^{-1} d V,} \\
& {\left[K_{q q}^{e}\right]=[X]^{-T} \int_{V} z^{2}\left[L_{K}\right]^{T}[D]\left[L_{K}\right][X]^{-1} d V,} \\
& {\left[K_{q \Phi}^{e}\right]=\left[K_{\Phi q}^{e}\right]^{T}=-[X]^{-T} \int_{V} z\left[L_{K}\right]^{T}[e]^{T} B_{z} d V,} \\
& {\left[K_{q \Theta}^{e}\right]=\left[K_{\Theta q}^{e}\right]^{T}=-[X]^{-T} \int_{V} z\left[L_{K}\right]^{T}[\lambda]^{T} d V,} \\
& {\left[K_{\Phi \Phi}^{e}\right]=-\int_{V} B_{z}^{T}\left[\epsilon^{T}\right] B_{z} d V,} \\
& {\left[K_{\Phi \Theta}^{e}\right]=-\int_{V} B_{z}^{T}\left[p^{T}\right] d V,} \\
& \{\bar{f}\}=\int_{V}\left\{f_{v}\right\} d V+\int_{A}\left\{f_{A}\right\} d V, \\
& \left\{Q_{a}\right\}=\int_{A} \sigma_{q} d A .
\end{aligned}
$$


Considering that the variation of $\left\{q_{k}\right\}$ and $\{\Phi\}$ is arbitrary, we obtain two equilibrium equations rewritten in generalized coordinates for the $k$ th element:

$$
\begin{aligned}
& {\left[M_{q q}^{e}\right]\left\{\ddot{q}_{k}\right\}+\left[K_{q q}^{e}\right]\left\{q_{k}\right\}+\left[K_{q \Phi}^{e}\{\Phi\}\right]-\left[K_{\Theta \Phi}^{e}\{\Theta\}\right]} \\
& -\{\bar{f}\}=0 \\
& {\left[K_{\Phi q}^{e}\right]\left\{q_{k}\right\}+\left[K_{\Phi \Theta}^{e}\right]\{\Theta\}+\left[K_{\Phi \Phi}^{e}\right]\{\Phi\}+\left\{Q_{a}\right\}=0,}
\end{aligned}
$$

with $K_{q q}^{e}$ being the extended stiffness matrix and $M_{q q}^{e}$ the elementary mass matrix. By integrating $K_{q q}^{e}$ in the direction of $z$, we obtain

$$
\left[K_{q q}^{e}\right]=\sum_{i=1}^{3} h_{i}[X]^{-T} \int_{A}\left[L_{K}\right]^{T}\left[D_{i}\right]\left[L_{K}\right] d A[X]^{-1},
$$

where $h_{i}$ are given by

$$
\begin{aligned}
& h_{1}=h_{a}\left(\frac{h_{p}}{2}+\frac{h_{a}}{2}\right)^{2}+\frac{h_{a}^{3}}{12}, \\
& h_{2}=\frac{h_{p}^{3}}{12}, \\
& h_{3}=h_{c}\left(\frac{h_{p}}{2}+\frac{h_{c}}{2}\right)^{2}+\frac{h_{c}^{3}}{12},
\end{aligned}
$$

and $D_{a}, D_{p}$, and $D_{c}$, for $i=1,2,3$, are calculated from (4) for the thermopiezoelectric material and the plate, respectively, and $d A=d x d y$.

The integration in $z$ of the elementary mass matrix gives

$$
\left[M_{q q}^{e}\right]=\sum_{i=1}^{3} \rho[X]^{-T} \int_{A}\left[L_{M}\right]^{T}\left[H_{i}\right]\left[L_{M}\right] d A[X]^{-1},
$$

with $\rho_{1}=\rho_{a}, \rho_{2}=\rho_{p}$, and $\rho_{3}=\rho_{c}$ and $\left[H_{i}\right]($ for $i=1,2,3)$ are

$$
\begin{aligned}
& {\left[H_{1}\right]=\left[H_{a}\right]=\left[\begin{array}{ccc}
h_{a} & 0 & 0 \\
0 & h_{1} & 0 \\
0 & 0 & h_{1}
\end{array}\right],} \\
& {\left[H_{2}\right]=\left[H_{p}\right]=\left[\begin{array}{ccc}
h_{p} & 0 & 0 \\
0 & h_{2} & 0 \\
0 & 0 & h_{2}
\end{array}\right],} \\
& {\left[H_{3}\right]=\left[H_{c}\right]=\left[\begin{array}{ccc}
h_{c} & 0 & 0 \\
0 & h_{3} & 0 \\
0 & 0 & h_{3}
\end{array}\right] .}
\end{aligned}
$$

By integrating the direction $z$, the thermoelastic coupling matrix $\left[K_{q \Theta}^{e}\right]$ for the three materials is given by

$$
\left[K_{q \Theta}^{e}\right]=\sum_{i=1}^{3} h_{i}[X]^{-T} \int_{A}\left[L_{K}\right]^{T}\left[\lambda_{i}\right]\left[L_{K}\right] d A[X]^{-1},
$$

with $\lambda_{i}$ being the matrix of each thermoelastic layer given in Table 1. The elementary stiffness matrix is due to electromechanical coupling; $\left[K_{q \Phi}^{e}\right]$ and pyroelectric $\left[K_{\Theta \Phi}^{e}\right]$ are integrated along the direction $z$ with respect to the thickness of each thermopiezoelectric layer $\left(d V=d V_{a}\right.$ or $\left.d V_{c}\right)$. Thus,

$$
\begin{aligned}
& {\left[K_{q \Phi}^{e}\right]=-\frac{1}{2}\left(h_{p} h_{a}+h_{a}^{2}\right)[X]^{-T} \int_{A}\left[L_{K}\right]^{T}[e]_{a}^{T} B_{z} d A,} \\
& {\left[K_{\Phi \Phi}^{e}\right]_{a}=-\frac{4 a b}{h_{a}}\left[\epsilon_{a}^{S}\right],} \\
& {\left[K_{q \Phi}^{e}\right]=\frac{1}{2}\left(h_{p} h_{c}+h_{c}^{2}\right)[X]^{-T} \int_{A}\left[L_{K}\right]^{T}[e]_{c}^{T} B_{z} d A,} \\
& {\left[K_{\Phi \Phi}^{e}\right]_{c}=-\frac{4 a b}{h_{c}}\left[\epsilon_{c}^{S}\right],} \\
& {\left[K_{\Theta \Phi}^{e}\right]_{a}} \\
& \quad=-\frac{1}{2}\left(h_{p} h_{a}+h_{a}^{2}\right)[X]^{-T} \int_{A}\left[L_{K}\right]^{T}[p]_{a}^{T} B_{z} d A, \\
& {\left[K_{\Theta \Phi}^{e}\right]_{c}=\frac{1}{2}\left(h_{p} h_{c}+h_{c}^{2}\right)[X]^{-T} \int_{A}\left[L_{K}\right]^{T}[p]_{c}^{T} B_{z} d A .}
\end{aligned}
$$

Equations (41), (43), (46), and (48) are integrated numerically using the quadratic integration method [11] as follows:

$$
\begin{aligned}
& {\left[K_{q q}^{e}\right]} \\
& =\sum_{i=1}^{3} h_{i}[X]^{-T} \sum_{\eta} \sum_{\xi}\left[L_{K}\right]^{T}\left[D_{i}\right]\left[L_{K}\right] W_{\eta} W_{\xi}[X]^{-1}, \\
& {\left[M_{q q}^{e}\right]} \\
& =\sum_{i=1}^{3} \rho_{i}[X]^{-T} \sum_{\eta} \sum_{\xi}\left[L_{M}\right]^{T}\left[H_{i}\right]\left[L_{M}\right] W_{\eta} W_{\xi}[X]^{-1}, \\
& {\left[K_{q \Theta}^{e}\right]} \\
& =\sum_{i=1}^{3} \rho_{i}[X]^{-T} \sum_{\eta} \sum_{\xi}\left[L_{M}\right]^{T}\left[\lambda_{i}\right]\left[L_{M}\right] W_{\eta} W_{\xi}[X]^{-1}, \\
& {\left[K_{q \Phi}^{e}\right]_{a}=h^{a}[X]^{-T} \sum_{\eta} \sum_{\xi}\left[L_{K}\right]^{T}[e]_{a}^{T}\left[L_{K}\right] W_{\eta} W_{\xi} B_{z},} \\
& {\left[K_{q \Phi}^{e}\right]_{c}=h^{c}[X]^{-T} \sum_{\eta} \sum_{\xi}\left[L_{K}\right]^{T}[e]_{c}^{T}\left[L_{K}\right] W_{\eta} W_{\xi} B_{z},} \\
& {\left[K_{\Theta \Phi}^{e}\right]=h^{a}[X]^{-T} \sum_{\eta} \sum_{\xi}\left[L_{K}\right]^{T}[p]_{a}^{T}\left[L_{K}\right] W_{\eta} W_{\xi} B_{z},} \\
& {\left[K_{\Theta \Phi}^{e}\right]=h^{c}[X]^{-T} \sum_{\eta} \sum_{\xi}\left[L_{K}\right]^{T}[p]_{c}^{T}\left[L_{K}\right] W_{\eta} W_{\xi} B_{z},}
\end{aligned}
$$

where $(\xi, \eta)$ are the coordinates of Gaussian point integration and $W_{\xi}, W_{\eta}$ are the associated weight factors with $h^{a}=$ $-(1 / 2)\left(h_{p} h_{a}+h_{a}^{2}\right)$ and $h^{c}=(1 / 2)\left(h_{p} h_{c}+h_{c}^{2}\right)$. 
All elementary matrices constructed may be assembled into global matrices as follows:

$$
\begin{gathered}
{[M]=\sum_{k=1}^{N}\left[T_{k}\right]^{T}\left[M_{q q}^{e}\right]\left[T_{k}\right],} \\
{\left[K_{q q}\right]=\sum_{k=1}^{N}\left[T_{k}\right]^{T}\left[K_{q q}^{e}\right]\left[T_{k}\right],}
\end{gathered}
$$

where $N$ is the number of finite elements and $T_{k}$ is a distribution matrix defined by

$$
T_{k}(i, j)= \begin{cases}0 & \text { if } j \neq m_{k}(i) \\ 1 & \text { if } j=m_{k}(i)\end{cases}
$$

for $i=1,2, \ldots, 12$ and $j=1,2, \ldots, n_{\text {dof }}$ with (dof) being the number of degrees of freedom of the entire structure. $m_{k}$ is the indicator vector containing the degrees of freedom 3(dof) of $n$th node (1, 2, 3, or 4; Figure 1) in $k$ th finite element given by

$$
m_{k}=\left\{3 n_{k}-23 n_{k}-13 n_{k}\right\} .
$$

Considering that there are $n_{a}$ actuators and $n_{c}$ sensors distributed on the surface of the plate, (40) including damping matrix $\left[C_{q q}\right]$ can be written in the following global shape:

$$
\begin{gathered}
{[M]\{\ddot{g}\}+\left[C_{q q}\right]\{\dot{g}\}+\left[K_{q q}\right]\{g\}+\sum_{k=1}^{n_{e i}}\left[T_{k}\right]_{i}^{T}} \\
\cdot\left(\left[K_{q \Phi}\right]_{i}\{\Phi\}-\left[K_{q \Theta}\right]\{\Theta\}\right)-\{F\}=0, \\
\sum_{k=1}^{n_{e i}}\left(\left[K_{\Phi q}\right]_{i}\left[T_{k}\right]_{i}\{g\}+\left[K_{\Phi \Theta}\right]_{i}\left[T_{k}\right]_{i}\{\Theta\}\right. \\
\left.+\left[K_{\Phi \Phi}\right]_{i}\{\Phi\}+Q_{a}\right)=0
\end{gathered}
$$

where $\left[T_{k}\right]_{i}$ is the distribution matrix indicating the position of the $k$ th element on the plate and is composed of numbers 1 or 0 . The figure 0 means that no thermopiezoelectric element (actuator or sensor) is present while the figure 1 indicates the presence of the element. $n_{e i}$ is the number of finite elements of $i$ th thermopiezoelectric actuator/sensor and $\{g\}$ is the vector of nodal displacement of the entire structure.

We have no voltage applied to the sensor $\left(Q_{a}=0\right)$, so the electrical potential generated is calculated through (55) for $i=1,2, \ldots, n_{s}$ as follows:

$$
\begin{aligned}
& \left\{\Phi_{c}\right\}=-\sum_{k=1}^{n_{e i}}\left[K_{\Phi \Phi}\right]_{c i}^{-1} \\
& \cdot\left(\left[K_{\Phi q}\right]_{c i}\left[T_{k}\right]_{i}\{g\}+\left[K_{\Phi \Theta}\right]_{c i}\left[T_{k}\right]_{i}\{\Theta\}\right)=0 .
\end{aligned}
$$

The total voltage $\Phi$ is composed of tension $\Phi_{s}$ sensed by the sensor, tension $\Phi_{s a}$ sensed by the actuator, and finally the applied voltage $\Phi_{a}$. So, the total voltage $\Phi$ is given by

$$
\{\Phi\}=\left\{\Phi_{s}\right\}+\left\{\Phi_{s a}\right\}+\left\{\Phi_{a}\right\} .
$$

The global dynamic equation can be formed by substitution of (56) into (57) and then into (54). The introduction of electrical forces due to the actuator forces, pyroelectric thermoelastic forces, and external mechanical forces leads to $\left(j=1,2, \ldots, n_{a}\right)$

$$
\begin{aligned}
{[M]\{\ddot{g}\}+\left[K_{q q}^{*}\right]\{g\}=} & \{F\}+\left\{F_{\text {el }}\right\}_{j}+\left\{F_{\text {eth }}\right\} \\
& +\left\{F_{\text {pth }}\right\}_{j},
\end{aligned}
$$

where $\left\{F_{\text {eth }}\right\}$ and $\left\{F_{\text {pth }}\right\}_{j}$ are the thermoelastic and pyroelectric forces, respectively, given by

$$
\begin{aligned}
\left\{F_{\text {eth }}\right\} & =\left[K_{q \Theta}\right]\{\Theta\}, \\
\left\{F_{\text {pth }}\right\}_{j} & =-\left[K_{q \Phi}\right]\left[K_{\Phi \Phi}\right]^{-1}\left[K_{\Phi \Theta}\right]\{\Theta\},
\end{aligned}
$$

and $\left[K_{q q}^{*}\right]$ and $\left\{F_{\mathrm{el}}\right\}$ (electrical force) are given, respectively, by

$$
\begin{aligned}
& {\left[K_{q q}^{*}\right]=\left[K_{q q}\right]-\left[K_{\mathrm{el}}\right],} \\
& \left\{F_{\mathrm{el}}\right\}_{j}=-\sum_{i=1}^{n_{e j}}\left[T_{k}\right]_{j}^{T}\left[K_{q \Phi}\right]_{a j},
\end{aligned}
$$

for $j=1,2, \ldots, n_{a}$. The electric stiffness matrix $\left[K_{\mathrm{el}}\right]$ can be written as

$$
\begin{aligned}
& \left\{K_{\mathrm{el}}\right\} \\
& =\sum_{i=1}^{n_{c}} \sum_{k s=1}^{n_{e i}}\left[T_{k}\right]_{i}^{T}\left[K_{q \Phi}\right]_{c i}\left[K_{\Phi \Phi}\right]_{c i}^{-1}\left[K_{\Phi q}\right]_{c i}\left[T_{k}\right]_{i} \\
& \quad+\sum_{j=1}^{n_{a}} \sum_{k=1}^{n_{e i}}\left[T_{k}\right]_{j}^{T}\left[K_{q \Phi}\right]_{a j}\left[K_{\Phi \Phi}\right]_{a j}^{-1}\left[K_{\Phi q}\right]_{a j}\left[T_{k}\right]_{j} .
\end{aligned}
$$

The static equation can be written using (33) as follows:

$$
\{\zeta\}=\left[K_{q q}^{*}\right]^{-1}\left(\{F\}+\left\{F_{\text {el }}\right\}+\left\{F_{\text {eth }}\right\}+\left\{F_{\text {pth }}\right\}\right) .
$$

For the transformation into a state space model, equations of motion can be written as

$$
\begin{aligned}
& \{\dot{\mathbf{x}}\}=[\mathbf{A}]\{\mathbf{x}\}+[\mathbf{B}]\left\{\Phi_{a}\right\}+\left[\mathbf{B}^{\text {pert }}\right]\left\{u_{\text {pert }}\right\}+\left[\mathbf{B}^{\text {th }}\right], \\
& \{\mathbf{y}\}=[\mathbf{C}]\{\mathbf{x}\},
\end{aligned}
$$




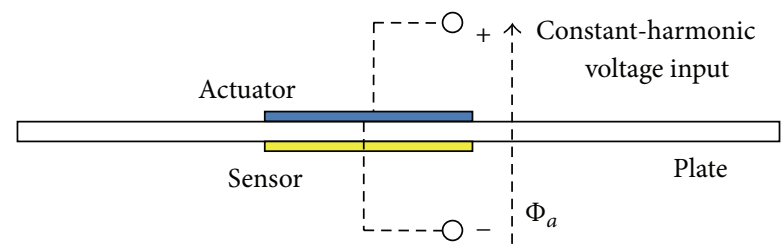

FIGURE 2: Actuation by application of constants or harmonic tensions.

where $[\mathbf{A}]$ is the matrix structure, $[\mathbf{B}]$ is the control matrix, $\left[\mathbf{B}^{\text {pert }}\right]$ is the matrix of disturbances, and $\left[\mathbf{B}^{\text {th }}\right]$ is the thermal disturbance, given, respectively, by

$$
\begin{aligned}
{[\mathbf{A}] } & =\left[\begin{array}{c}
-[\bar{M}]^{-1}\left[\overline{C_{q q}}\right] \\
{[I]} \\
[1] \bar{M}]^{-1}\left[\overline{K_{q q}}\right]
\end{array}\right], \\
{[\mathbf{B}] } & =\left[\begin{array}{c}
\left.-[\bar{M}]^{-1}\left[\bar{K}_{q \phi}\right]\right], \\
{[0]}
\end{array}\right] \\
{\left[\mathbf{B}^{\text {pert }}\right] } & =\left[\begin{array}{c}
\left.[\bar{M}]^{-1}\{\bar{F}\}\right], \\
{[0]}
\end{array}\right] \\
{\left[\mathbf{B}^{\text {th }}\right] } & =\left[\begin{array}{c}
{[\bar{M}]^{-1}\left\{\left\{\bar{F}_{\text {eth }}\right\}+\left\{\bar{F}_{\text {pth }}\right\}\right\}} \\
{[0]}
\end{array}\right] .
\end{aligned}
$$

\section{Results and Discussions}

Thermopiezoelastic behavior and the active control of cantilever aluminium plate are presented. The model considered is a flexible piezocomposite rectangular plate size $(0.6 \mathrm{~m} \times$ $0.4 \mathrm{~m} \times 0.001 \mathrm{~m}$ ) whose geometric and physical properties are shown in Table 1 and in which two piezoelectric layers (size $0.25 \mathrm{~m} \times 0.25 \mathrm{~m} \times 0.0002 \mathrm{~m}$ ) are perfectly bonded (Figure 2 ). In the finite element modeling, the plate is divided into $60 \times$ 40 finite elements of equal size and each element has three mechanical, one electrical, and one temperature dofs. The study consists of three phases; the first is to verify the actuation in the static case by exciting the actuator by constant and harmonic inputs; the second phase corresponds to analyse the responses of the structure dynamics under a center plate pulse and study the effect of the location of piezoelectric elements on the effectiveness of control; and the third phase was dedicated to analyzing the effect of thermoelastic and pyroelectric coupling, by applying thermal gradient, on the dynamics of the structure and the control procedure.

3.1. Case of Absence of Thermal Effect. To validate the mathematical formulation and the finite element model in the static case, the configuration shown in Figure 2 gives an illustration of the mode of actuation of the actuator(s) bonded on the surface of the plate. This allows us to check the actuation states of the actuators and their acting on the plate. To this end, we apply a voltage $\bar{\Phi}_{a}$ (constant or harmonic) at the surfaces of the actuators causing a vibration plate.
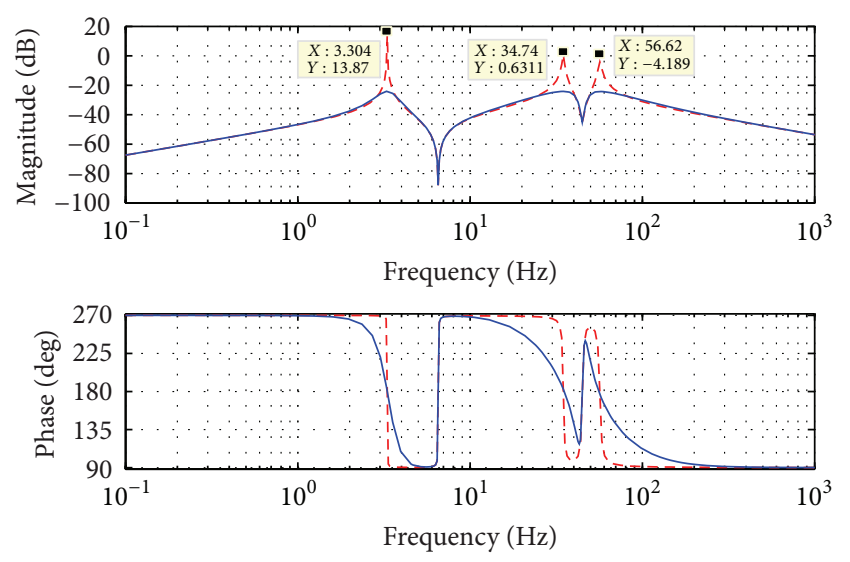

- - - Noncontrolled

FIGURE 3: Spectra of first modes 11, 12, and 21 of plate dynamics in open and closed loop. Mode 11 at $3.31 \mathrm{~Hz}$, mode 12 at $34.7 \mathrm{~Hz}$, and mode 21 at $56.4 \mathrm{~Hz}$.

We have given in Figure 3 the spectra of the three first modes 11, 12, and 21 and in Figure 4, for illustration, some examples of the first modes shape of vibration of the plate, that is, modes 11, 22, 33, 44, 12, 21, 13, and 14. Figure 5 shows the response to the actuator excitations by tensions with constant or different amplitudes. Figure 6 illustrates the state of the plate after a harmonic input voltage whose amplitudes and frequencies are chosen arbitrarily.

In order to verify the characteristics of the actuators, and by varying their size and position, and applying the two types of voltage excitations, it was shown that the plate can generate dynamics which can be controllable. In Figure 7, the symmetrical distributed pairs of patches (actuator/sensor) in several positions and with different sizes on both surfaces of the plate are placed. The center plate displacement in mode 11 with the corresponding actuator voltage and sensor output is shown in Figures 8 and 9. The LQR-Kalman method parameters (weight matrices $Q$ and $R$ with the gain control $G_{c}$ ) are taken equal to $10^{6}, 1$, and 200, respectively. Figure 10 gives the first modes of plate vibration with and without control. Moreover, we conclude that the control can be applied during the vibration of the plate without diminishing its quality or effectiveness as shown in Figure 11. The control is applied 0.5 seconds after the start of the oscillation. We note that the plate is quickly damped and consequently the actuator input tension begins to decrease after the application of control. In another side, the position and size of piezoelectric patches may increase or decrease the quality control. Indeed, we deduce from Figure 12 that the wider and nearer to the center of plate the piezoelectric patches are (point undergoing large deformations), the better the structure is controlled. This is justified by the fact that the sensor (mounted in front with the actuator) captures the highest deformations in the plate vibration so that the patches near to the sides of the plate capture the low vibration amplitudes, which affects the output voltage sensor, and therefore the responses of the actuator would be less effective. This is explained by the fact 
Mode $(1,1)$


Mode (2, 2)
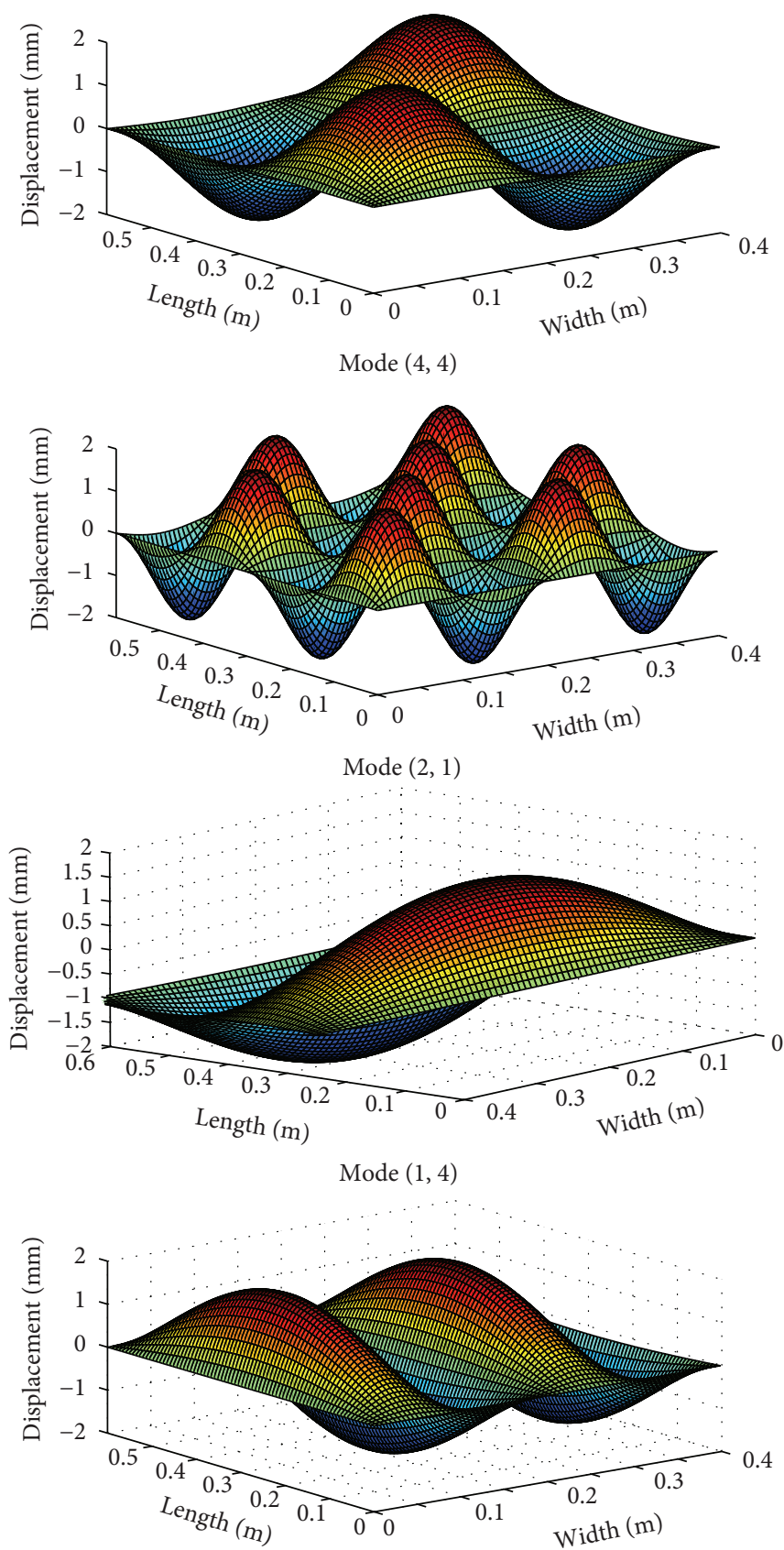

FIGURE 4: First modes shape of a plate drawn in 3D.

that the patches must be placed as possible near to the the pulse application point. However, if external perturbations are unpredictable, trials must be established to obtain the best positions to the control.

3.2. Case of Presence of the Thermal Effect. We assume at first that the plate and the layers thermopiezoelectric actuator/sensor are placed in a thermal field and the temperature of the plate reached quickly the studied case. From (23), the change in temperature can generate a voltage in the thermopiezoelectric sensor and the mechanical deformation created under the effect of temperature also produces an electrical signal in the sensor. In order to verify these changes in the deformation, we apply successively, in static case, a thermal gradient in the surface of the plate so that the top surface is hotter than the bottom surface. Because of this gradient, the centerline of the plate is deformed upward. Figure 13 gives the deformation of the plate for different values of the gradient of temperature and illustrates the thermoelastic effect. We recall that the sensor must be more 

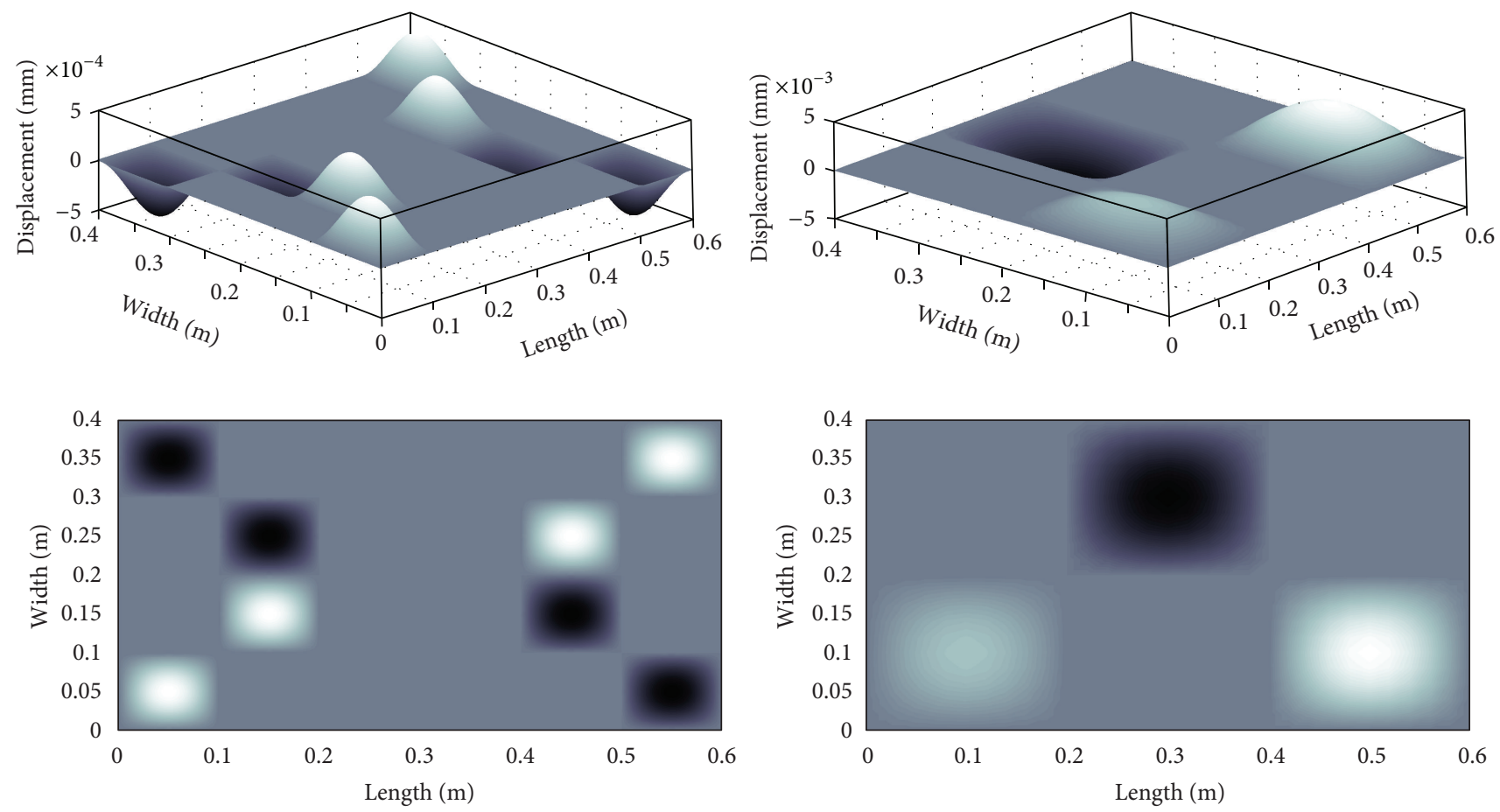

FIGURE 5: Static test of the used finite element and control methods by giving to the actuator in different sizes pulses with different amplitudes.
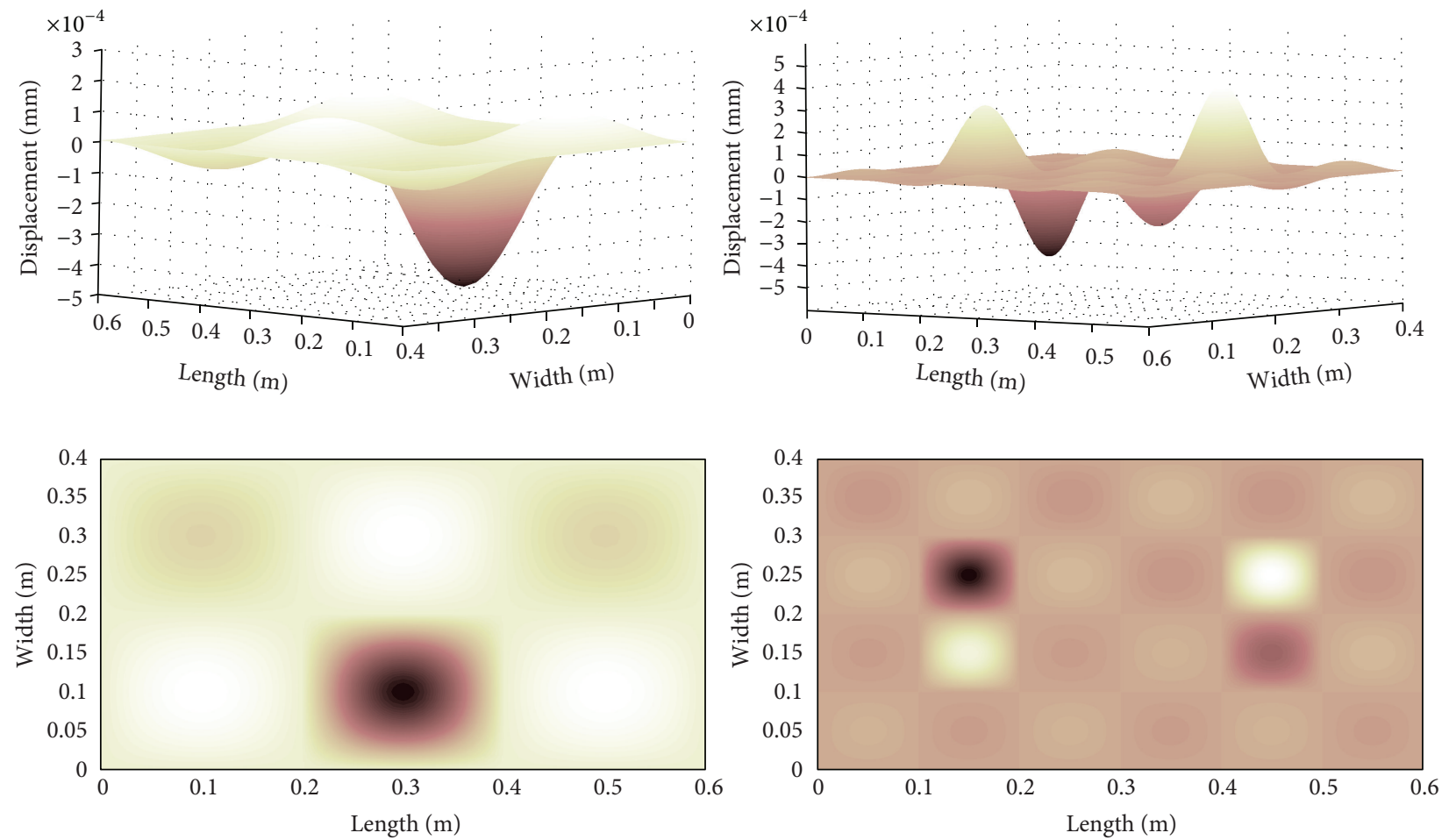

FIgURE 6: Plate dynamics under actuator harmonic input with change in position and size of actuator patches.

sensitive than the actuator (the coefficient of rigidity of the actuator must be higher compared to the sensor; see Table 1).

We apply, now, at the center of the flexible plate a pulse with amplitude $2 \mathrm{~N}$. After a period of 0.5 seconds of applying the impulse, a control based on the method LQG-Kalman is applied. The thermal effect due to a temperature gradient of $4^{\circ} \mathrm{C}$ is considered in this case (Figure 14). The weight matrices $Q$ and $R$ and gain control $G_{c}$ are taken equal to $10^{10}, 1$, and 200 , respectively. We assume that the material with which the piezoelectric patches are bonded has a good resistance to temperature change (the patches remain perfectly bonded to the surfaces of the plate). Figure 15 illustrates the compensation 


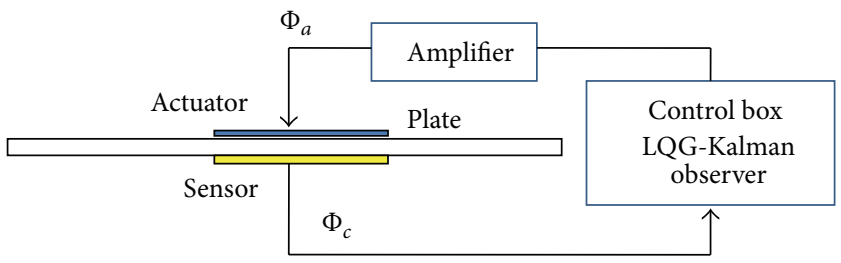

FIGURE 7: Closed loop of the control (sensor-controller-actuator) used in simulation.
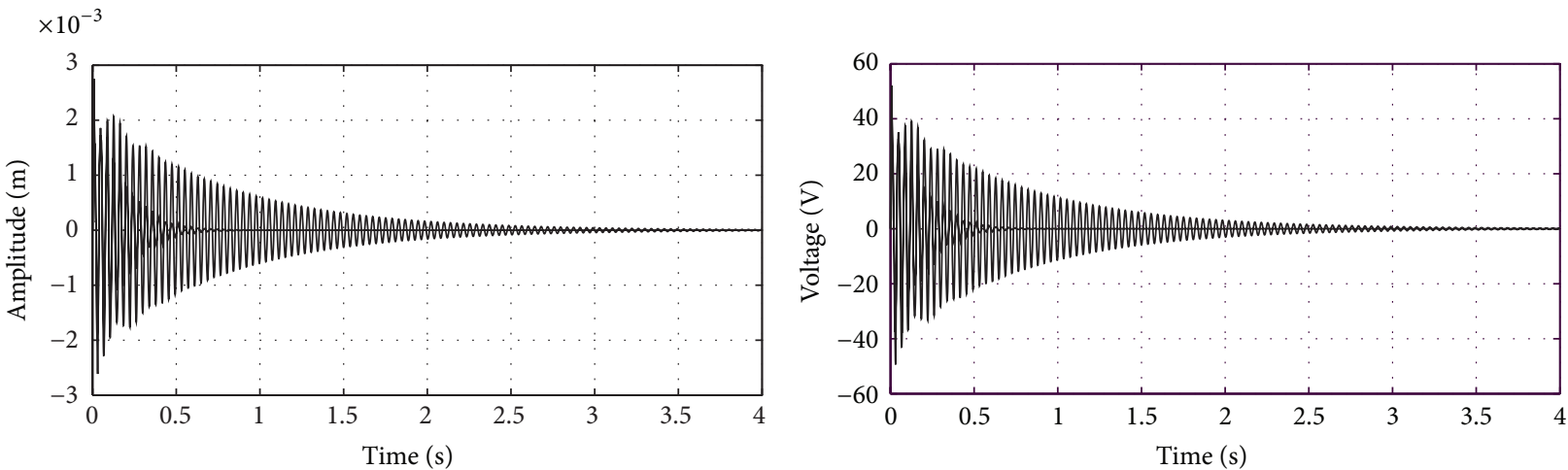

FIGURE 8: Response of a pulse $(3 \mathrm{~N})$ of displacement of center of plate and corresponding actuator input voltage $\left(Q=10^{6}, R=1\right.$, and $\left.G_{c}=200\right)$.


FIGURE 9: Response of sensor output voltage in open and closed loop $\left(Q=10^{6}, R=1\right.$, and $\left.G_{c}=200\right)$.
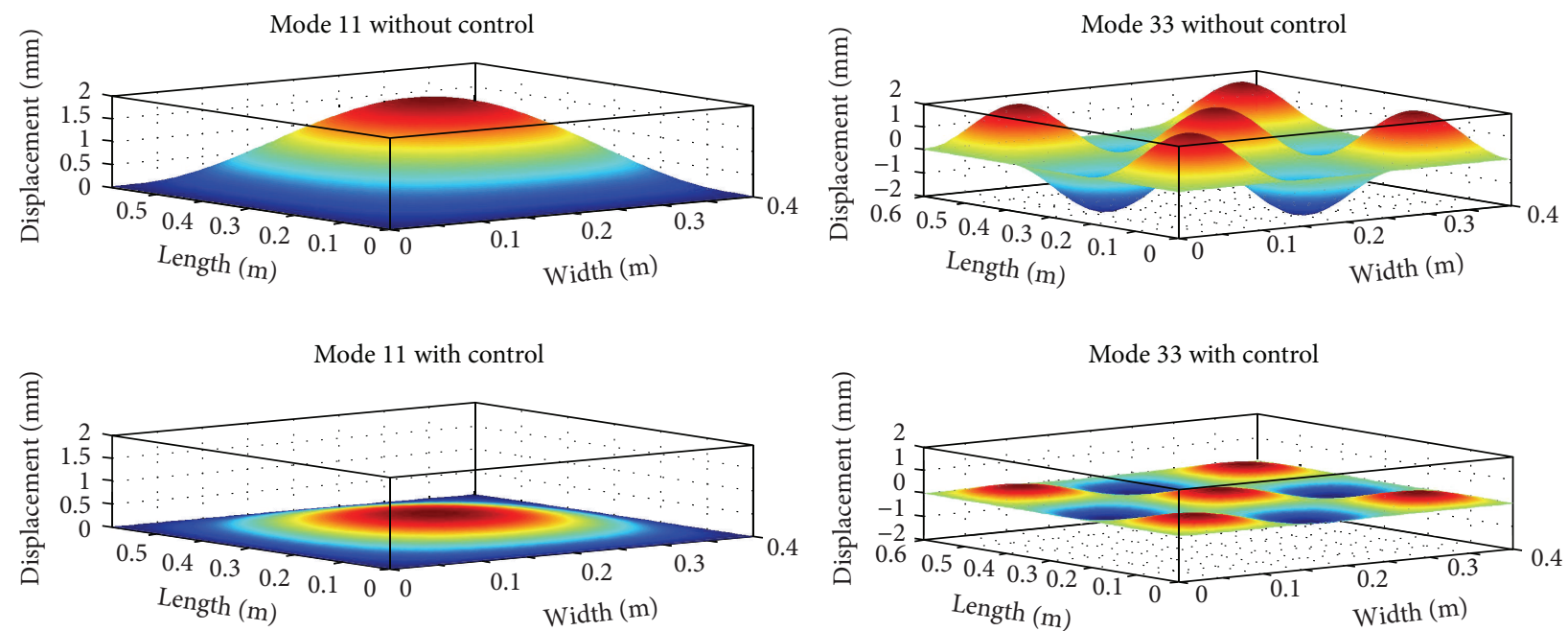

FIGURE 10: Shape of first modes 11 and 33 with and without control in $3 \mathrm{D}\left(Q=10^{6}, R=1\right.$, and $\left.G_{c}=200\right)$. 

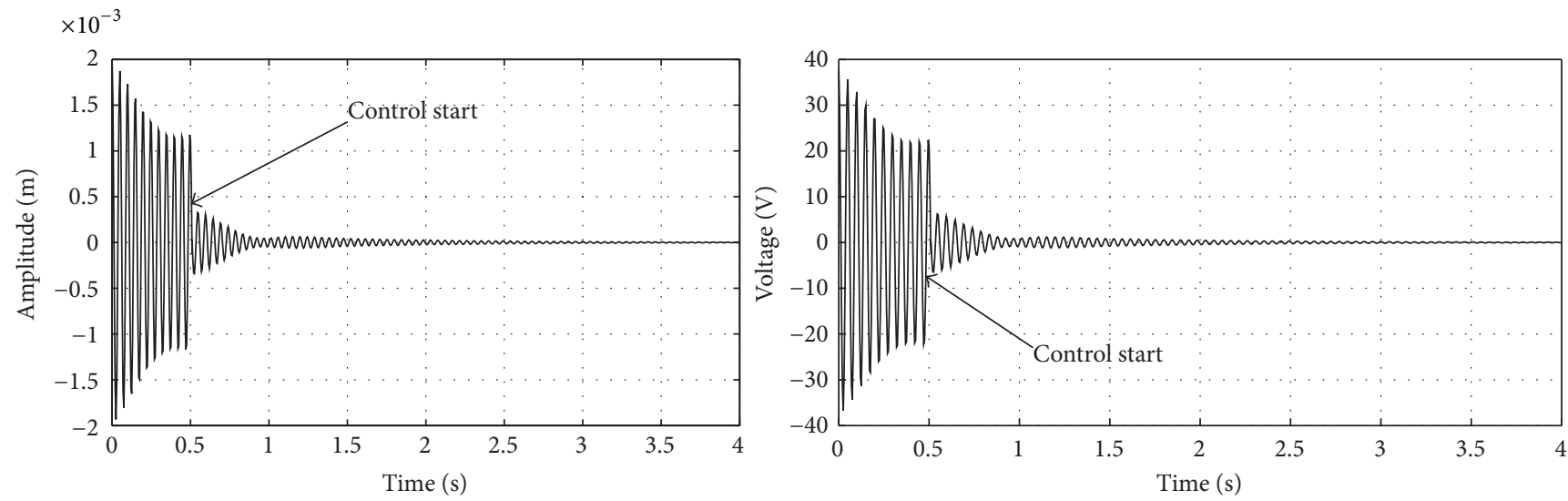

Figure 11: Application of control after 0.5 seconds from the vibration beginning $\left(Q=10^{6}, R=1\right.$, and $\left.G_{c}=200\right)$.
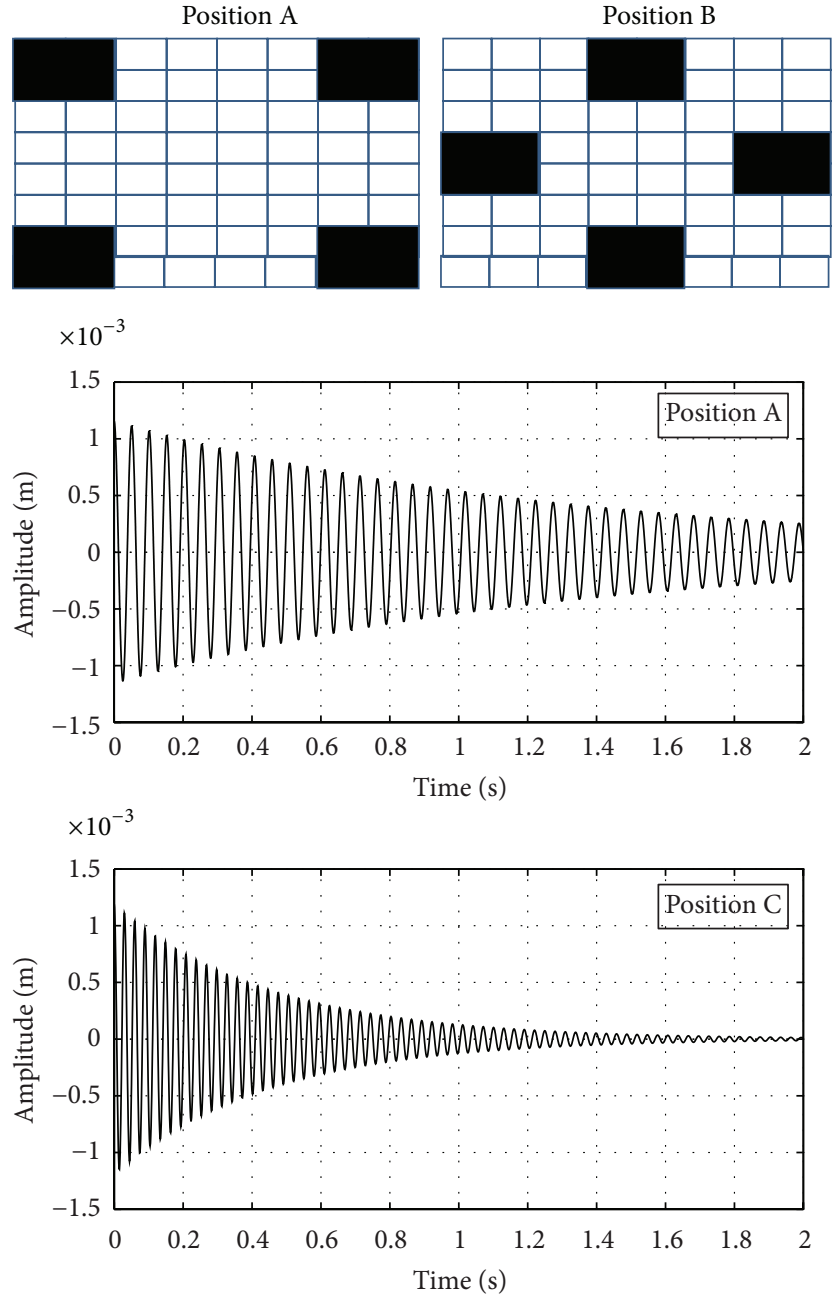
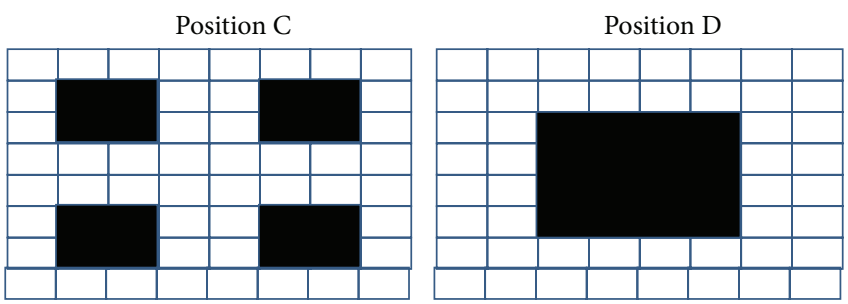

$\times 10^{-3}$
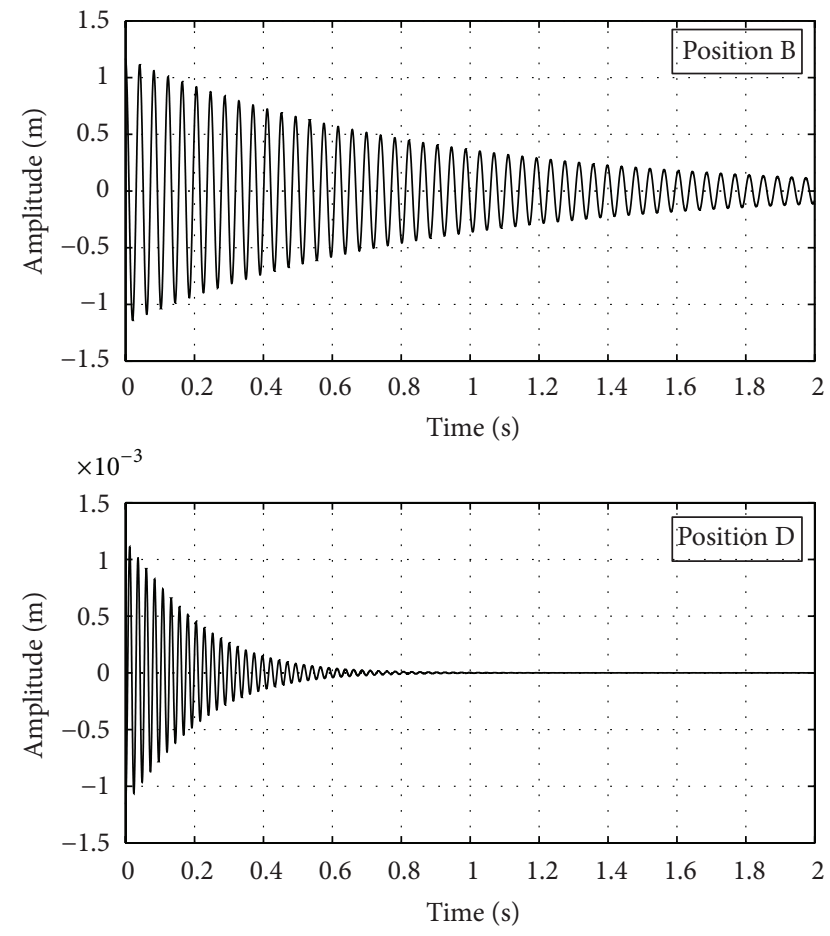

FIGURE 12: Influence of the position distribution of actuators and sensors on the quality of control $\left(Q=10^{6}, R=1\right.$, and $\left.G_{c}=200\right)$.

due to the application of the gradient without control. The amplitudes of vibration of the neutral plane centre stabilize at the amplitude $-2.5 \mathrm{~mm}$. This is logical because the temperature above the plate is high compared to that below. The plate continues to oscillate in the same amplitude. Consequently, the tension in the actuator increases at the application of the gradient due to the increased mechanical deformations by thermal expansion in the sensor and also the tension due to pyroelectric effect. Figure 15 gives also the corresponding actuator voltage that increases when the gradient is applied. 


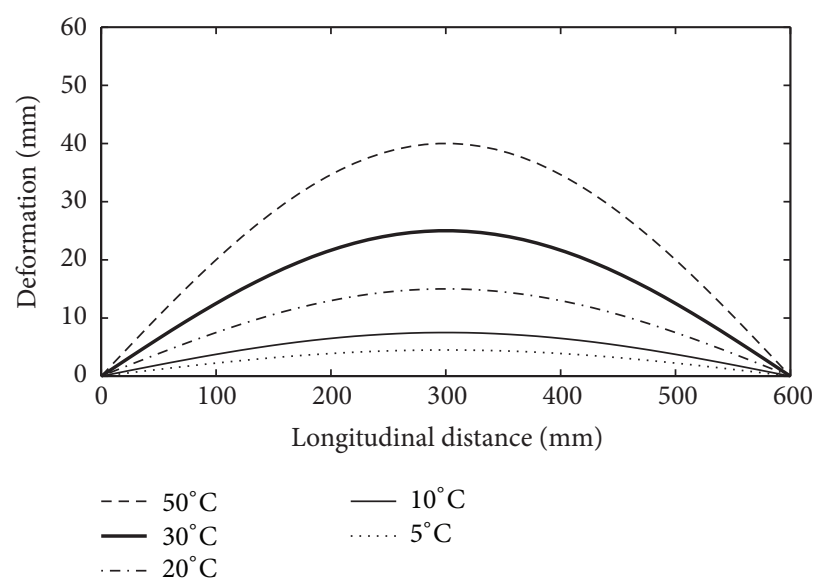

FIGURE 13: Plate neutral plan deformation for different values of temperature.

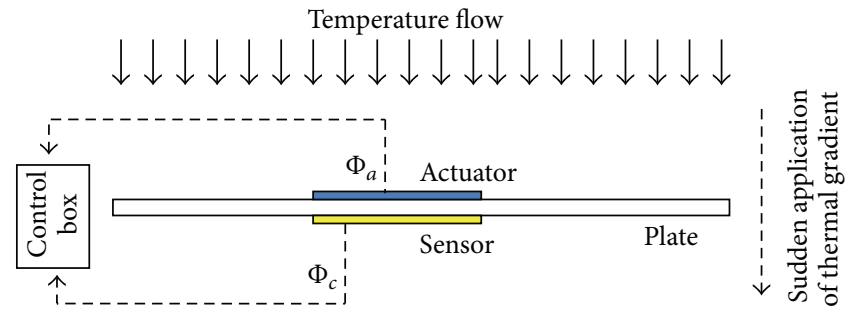

FIGURE 14: Application of a thermal gradient between surfaces of the plate.
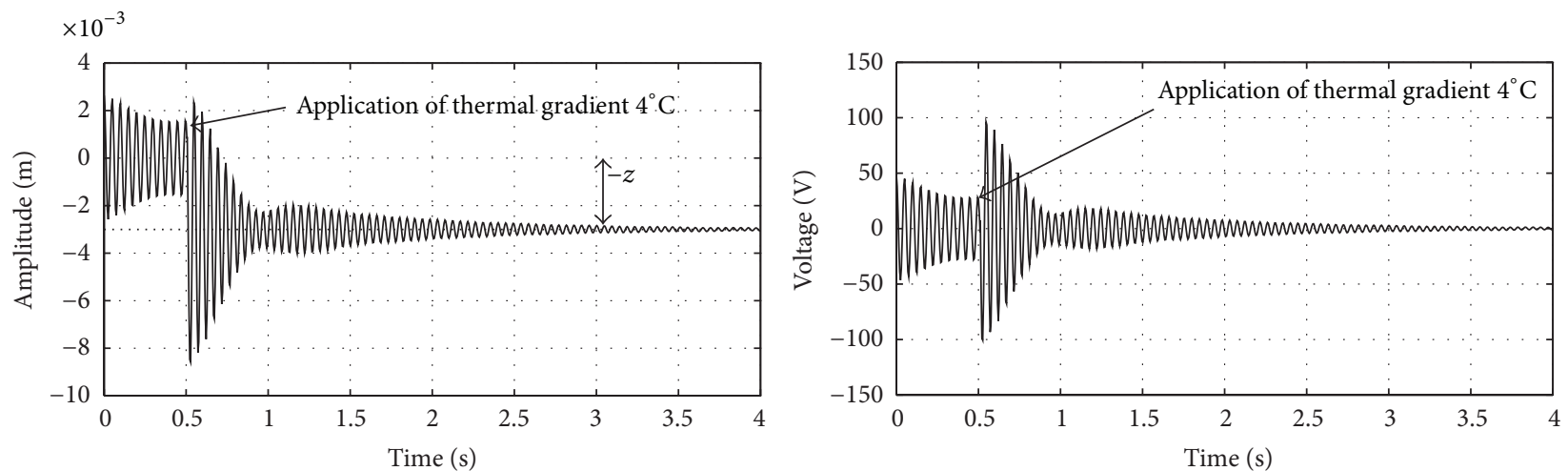

Figure 15: Displacement and the corresponding actuator input voltage of plate center and the actuator tension after the application of a $4^{\circ} \mathrm{C}$ thermal gradient.

We take the same position as before, and we apply the control (Figure 16). We note that the plate is checked just to the deviation amplitude $(-2.5 \mathrm{~mm})$. The corresponding tension in the actuator is shown in Figure 16. The only precaution is to not let the gradient be applied for a long time; otherwise, we lose the controllability monitoring. It should be noted here that the tension needed to be applied to piezoelectric layers is very important to offset a small gradient of $4^{\circ} \mathrm{C}$, for example. In addition, excessively high voltages applied to the actuator must be avoided in order to not depolarize the thermopiezoelectric material (PZT). This means that thermal gradients applied must be limited by the used material of the actuator. We also note that the application of a constant gradient for a long period cannot be monitored or controlled by the LQG method, but a sudden applied gradient is controllable. This is one of the advantages of this method, since all the thermal disturbances are unpredictable. Figure 17 shows this effect. After a period of 0.5 seconds of applying the pulse $(2 \mathrm{~N})$, the plate is subjected suddenly to a temperature gradient of $4^{\circ} \mathrm{C}$. The control is applied a second later. The structure is controlled during and after the application of the gradient. This shows that the used control method is effective for sudden changes in temperature. The tension with which the actuator acts is due to the pyroelectric effect (by increasing the temperature of the actuator itself) and the amplified 

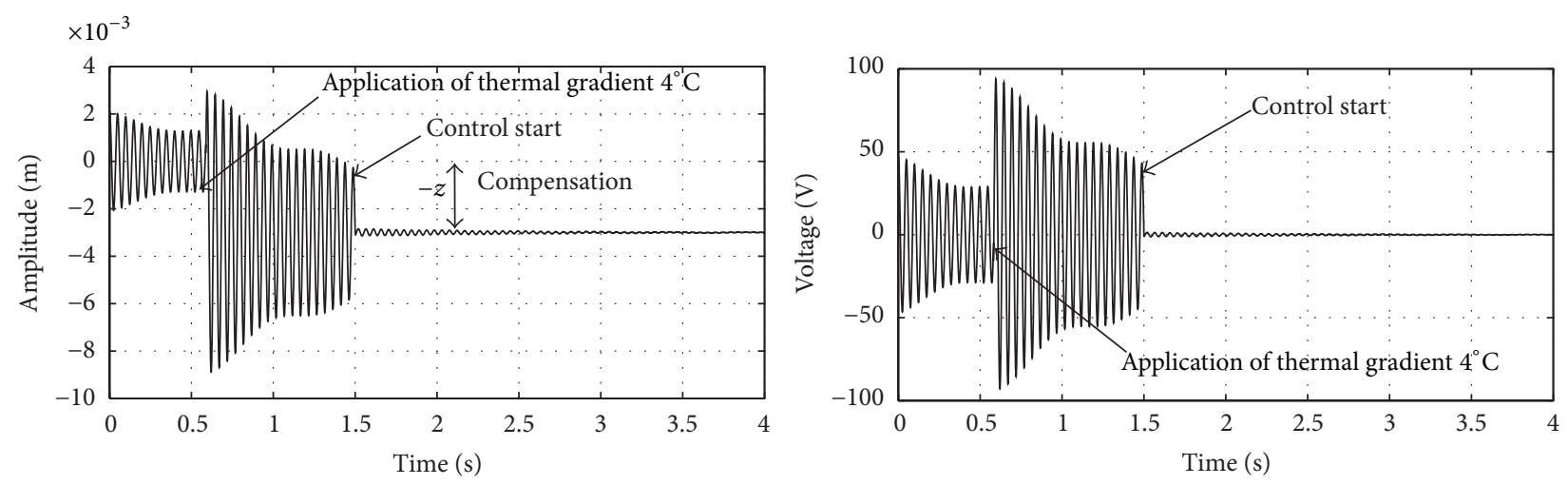

FIGURE 16: The noninfluence of the application of thermal gradient on control effectiveness $\left(Q=10^{10}, R=1\right.$, and $\left.G_{c}=200\right)$.
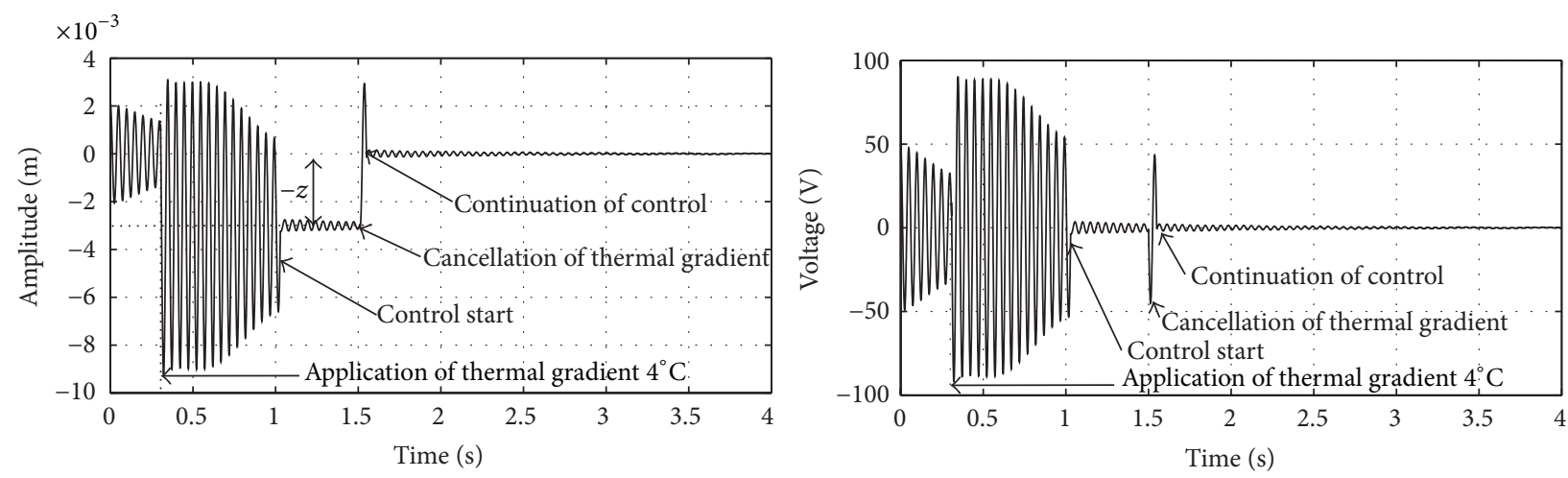

FIGURE 17: Application of sudden thermal gradient during the application of control $\left(Q=10^{10}, R=1\right.$, and $\left.G_{c}=200\right)$.

voltage from the controller. It receives a voltage sensor that is the result of the thermal effect (pyroelectric effect) and the effect of increasing mechanical and thermal deformations. The voltage then increases when a thermal gradient is applied and the intensity of control increases accordingly (Figure 17).

\section{Conclusion}

The modeling by the finite element method according to the theory of Kirchhoff of a rectangular cantilever piezocomposite plate with bonded thermopiezoelectric elements at its surfaces is presented and implemented. The optimal control based on the method of LQG-Kalman is applied and discussed. The influence of the location of the patches sensor/actuator and of the application of thermal gradient on the effectiveness of control is demonstrated. It is shown that the application of control during the vibration of the structure does not diminish the control quality; that is, the control can be applied at any time during the vibration of the plate. Moreover, the deformations produced by the sudden application of a thermal gradient can be controlled, to point out that the simulation programs diverge when an intense, or of long duration, thermal gradient is applied. Otherwise, this can infect the pyroelectric effect in the sensor or may deteriorate the polarization of the actuator.

\section{Conflict of Interests}

The authors declare that there is no conflict of interests regarding the publication of this paper.

\section{References}

[1] A. Görnandt and U. Gabbert, "Finite element analysis of thermopiezoelectric smart structures," Acta Mechanica, vol.154, no. 1-4, pp. 129-140, 2002.

[2] S. S. Vel and R. C. Batra, "Generalized plane strain thermopiezoelectric analysis of multilayered plates," Journal of Thermal Stresses, vol. 26, no. 4, pp. 353-377, 2003.

[3] G. L. C. M. de Abreu, J. F. Ribeiro, and V. Steffen Jr., "Finite element modeling of a plate with localized piezoelectric sensors and actuators," Journal of the Brazilian Society of Mechanical Sciences and Engineering, vol. 26, no. 2, pp. 117-128, 2004.

[4] J.-F. Deü and A. Benjeddou, "Free-vibration analysis of laminated plates with embedded shear-mode piezoceramic layers," International Journal of Solids and Structures, vol. 42, no. 7, pp. 2059-2088, 2005.

[5] N. Tanaka and T. Sanada, "Modal control of a rectangular plate using smart sensors and smart actuators," Smart Materials and Structures, vol. 16, no. 1, pp. 36-46, 2007.

[6] M. A. Trindade and A. Benjeddou, "Effective electromechanical coupling coefficients of piezoelectric adaptive structures: 
critical evaluation and optimization," Mechanics of Advanced Materials and Structures, vol. 16, no. 3, pp. 210-223, 2009.

[7] M. Sanbi, R. Saadani, K. Sbai, and M. Rahmoune, "Thermoelastic and pyroelectric couplings effects on dynamics and active control of smart piezolaminated beam modeled by finite element method," Smart Materials Research, vol. 2014, Article ID 145087, 10 pages, 2014.

[8] H.-J. Ding, F.-L. Guo, and P.-F. Hou, "A general solution for piezothermoelasticity of transversely isotropic piezoelectric materials and its applications," International Journal of Engineering Science, vol. 38, no. 13, pp. 1415-1440, 2000.

[9] J. Erhart, E. Kittinger, J. Privratska, and J. Tichy, Fundamentals of Piezoelectric Sensorics: Mechanical, Dielectric, and Thermodynamical Properties of Piezoelectric Materials, Springer, 2010.

[10] A. Erturk, Electromechanical modeling of piezoelectric energy harvesters [Ph.D. thesis], Virginia Polytechnic Institute and State University, 2009.

[11] K. J. Bathe, Finite Element Procedures in Engineering Analysis, Prentice-Hall, 1982.

[12] J. S. Yang and R. C. Batra, "Free vibrations of a linear thermopiezoelectric body," Journal of Thermal Stresses, vol. 18, no. 2, pp. 247-262, 1995.

[13] V. Lopes Jr., J. A. Pereira, and D. J. Inman, "Structural FRF acquisition via electric impedance measurement applied to damage location," in Proceedings of the 18th International Modal Analysis Conference (IMAC '00), European University Association (EUA), San Antonio, Tex, USA, February 2000. 

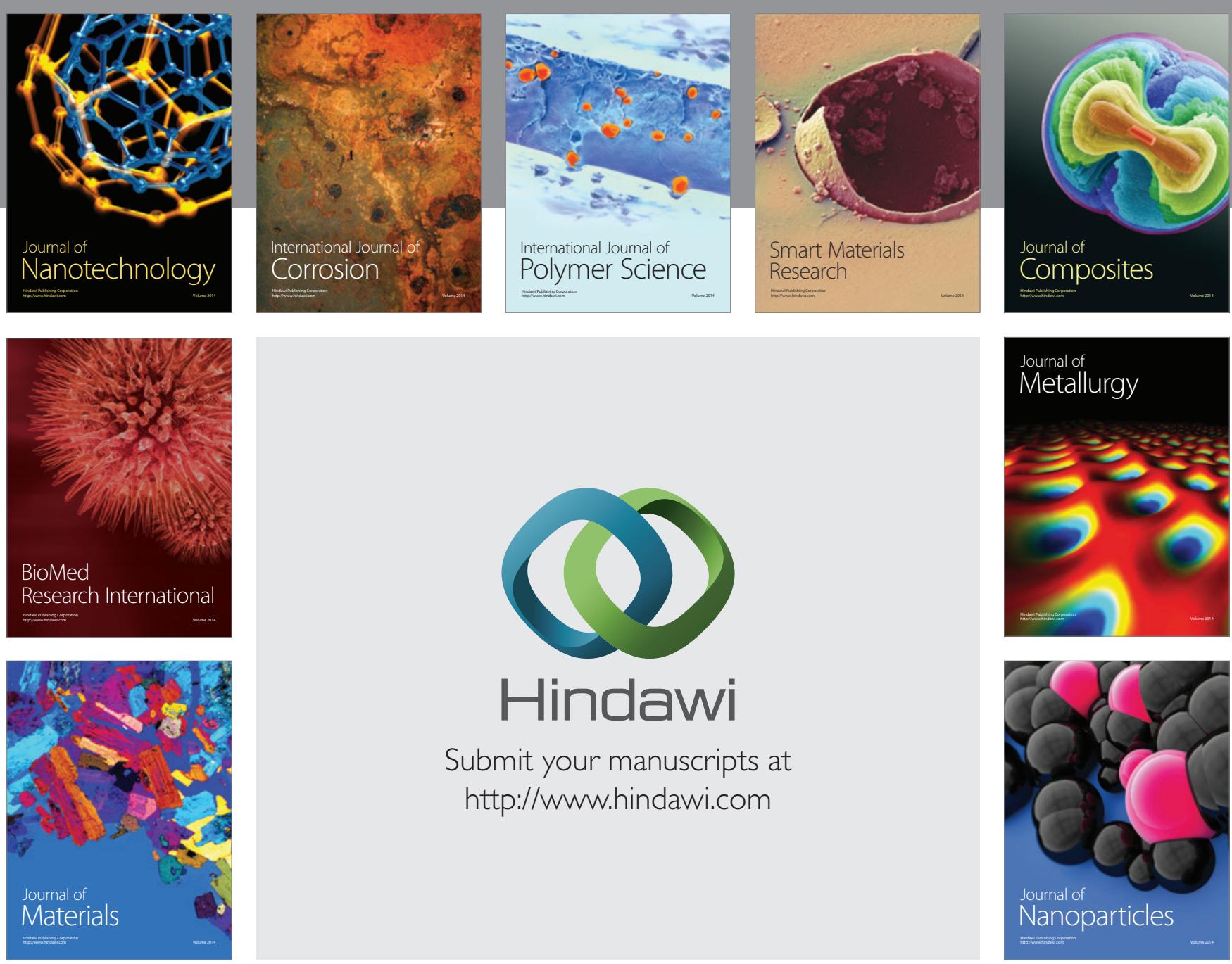

Submit your manuscripts at http://www.hindawi.com


\section{The Scientific World Journal}
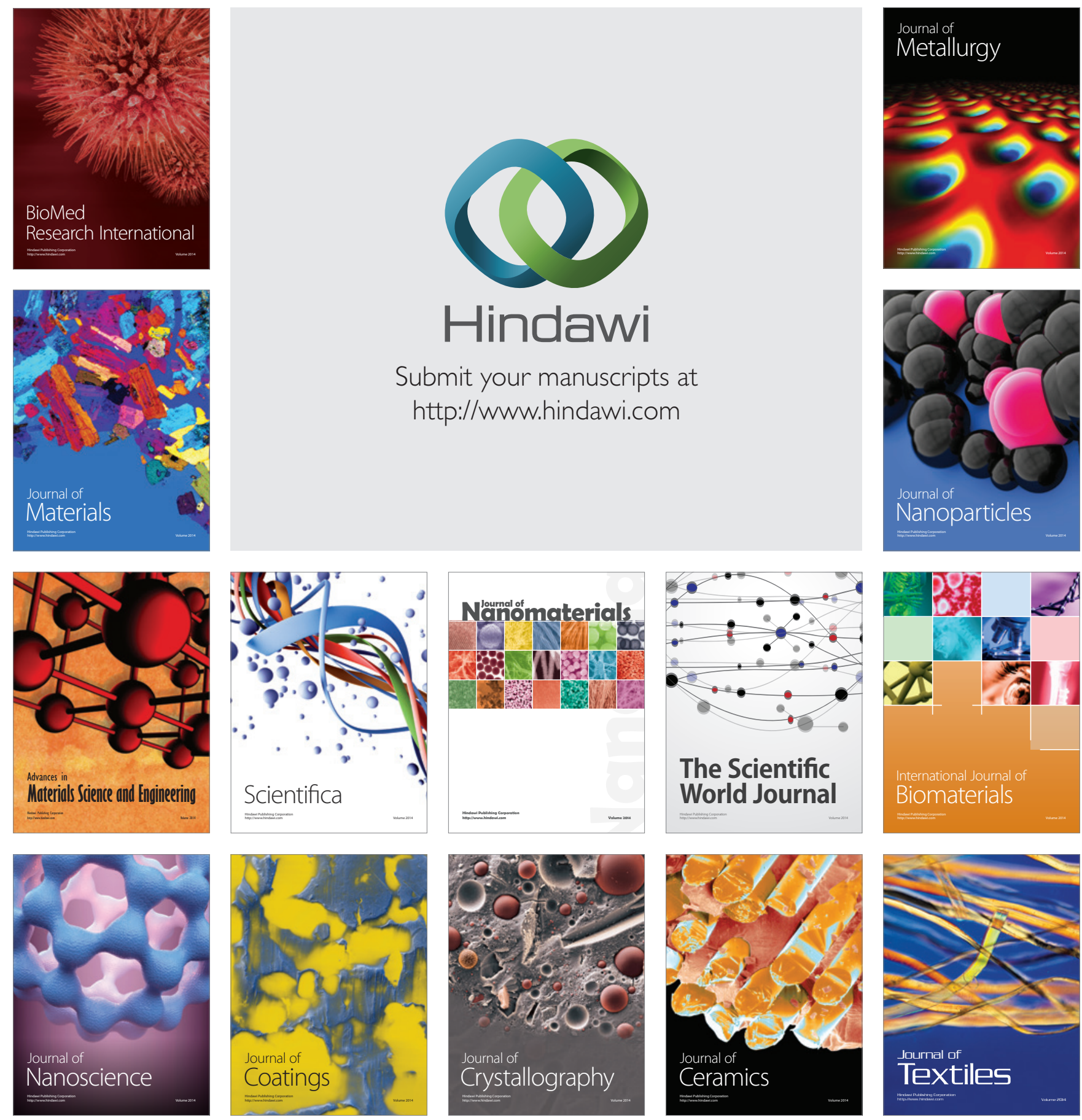